\title{
Experimental Testing of Advanced Scatter Search Designs for Global Optimization of Multimodal Functions
}

\author{
MANUEL LAGUNA $^{1}$ \\ Leeds School of Business, University of Colorado, Boulder, CO 80309-0419, USA \\ laguna@colorado.edu \\ RAFAEL MARTÍ ${ }^{2}$ \\ Departament D’Estadística i Investigació Operativa, Universitat de València, Burjassot 46100, Spain \\ rafael.marti@uv.es
}

Latest version: November 21, 2002

\begin{abstract}
Scatter search is an evolutionary method that, unlike genetic algorithms, operates on a small set of solutions and makes only limited use of randomization as a proxy for diversification when searching for a globally optimal solution. The scatter search framework is flexible, allowing the development of alternative implementations with varying degrees of sophistication. In this paper, we test the merit of several scatter search designs in the context of global optimization of multimodal functions. We compare these designs among themselves and choose one to compare against a well-known genetic algorithm that has been specifically developed for this class of problems. The testing is performed on a set of benchmark multimodal functions with known global minima.
\end{abstract}

\footnotetext{
${ }^{1}$ Partially supported by the visiting professor fellowship program of the University of Valencia (Grant Ref. No. 42743).

${ }^{2}$ Partially supported by the Ministerio de Ciencia y Tecnología of Spain: TIC2000-1750-C06-01.
} 


\section{Introduction}

Scatter search is a solution procedure that falls within the evolutionary framework and consists of five methods:

1. A Diversification Generation Method to generate a collection of diverse trial solutions, using one or more arbitrary trial solutions (or seed solutions) as an input.

2. An Improvement Method to transform a trial solution into one or more enhanced trial solutions. (Neither the input nor the output solutions are required to be feasible, though the output solutions are typically feasible. If the input trial solution is not improved as a result of the application of this method, the "enhanced" solution is considered to be the same as the input solution.)

3. A Reference Set Update Method to build and maintain a reference set consisting of the $b$ "best" solutions found (where the value of $b$ is typically small, e.g., no more than 20), organized to provide efficient accessing by other parts of the solution procedure. Several alternative criteria may be used to add solutions to the reference set and delete solutions from the reference set.

4. A Subset Generation Method to operate on the reference set, to produce a subset of its solutions as a basis for creating combined solutions. The most common subset generation method is to generate all pairs of reference solutions (i.e., all subsets of size 2).

5. A Solution Combination Method to transform a given subset of solutions produced by the Subset Generation Method into one or more combined solutions. The combination method is analogous to the crossover operator in genetic algorithms but it must be capable of combining two or more solutions.

The scatter search methodology is very flexible, since each of its elements can be implemented in a variety of ways and degrees of sophistication. Details about the origin and applications of scatter search can be found in, Glover (1998), Glover, Laguna and Martí (1999) and Laguna (2002). In our current development, we study the merit of alternative scatter search designs in the context of nonlinear optimization. In particular, we use a benchmark set of unconstrained problems, for which global optima are known. That is, the class of problems that we use for testing can be characterized as:

$$
\begin{array}{ll}
\text { Minimize } & f(x) \\
\text { Subject to } & l \leq x \leq u
\end{array}
$$

Where $f(x)$ is a nonlinear function and $x$ is a vector of continuous and bounded variables. We test several alternatives for generating diversification and updating the reference set in a procedure that does not use an improvement method and in which combinations are linear and limited to pairs of solutions. We also test the use of a two-phase intensification. These strategies are added to a basic procedure, which is outlined in form of pseudo-code in Figure 1.

The basic procedure in Figure 1 starts with the creation of an initial reference set of solutions (RefSet). The Diversification Generation Method is used to build a large set of diverse solutions $P$. The size of $P$ (PSize) is typically 10 times the size of RefSet. Initially, the reference set RefSet consists of $b$ distinct and maximally diverse solutions from $P$. The solutions in RefSet are ordered according to quality, where the best solution is the first one in the list. The search is then initiated by assigning the value of TRUE to the Boolean variable NewSolutions. In step 3, NewSubsets is constructed and NewSolutions is switched to FALSE. Since we are focusing our attention to subsets of size 2, the cardinality of NewSubsets 
corresponding to the initial reference set is given by $\left(b^{2}-b\right) / 2$, which accounts for all pairs of solutions in RefSet. The pairs in NewSubsets are selected one at a time in lexicographical order and the Solution Combination Method is applied to generate one or more solutions in step 5. If a newly created solution improves upon the worst solution currently in RefSet, the new solution replaces the worst and RefSet is reordered in step 6. The NewSolutions flag is switched to TRUE and the subset $s$ that was just combined is deleted from NewSubsets in steps 7 and 8, respectively.

1. Start with $P=\varnothing$. Use the Diversification Generation Method to construct a solution $x$. If $x \notin P$ then add $x$ to $P$ (i.e., $P=P \cup x$ ), otherwise, discard $x$. Repeat this step until $|P|=P$ Size. Build RefSet $=\left\{x^{1}, \ldots, x^{b}\right\}$ with $b$ diverse solutions in $P$.

2. Evaluate the solutions in RefSet and order them according to their objective function value such that $x^{1}$ is the best solution and $x^{b}$ the worst. Make NewSolutions $=$ TRUE.

while (NewSolutions) do

3. Generate NewSubsets, which consists of all pairs of solutions in RefSet that include at least one new solution. Make NewSolutions $=$ FALSE.

while $($ NewSubsets $\neq \varnothing)$ do

4. Select the next subset $s$ in NewSubSets.

5. Apply the Solution Combination Method to $s$ to obtain one or more new solutions $x$.

if $\left(x\right.$ is not in RefSet and $f(x)<f\left(x^{b}\right)$ ) then

6. Make $x^{b}=x$ and reorder RefSet.

7. Make NewSolutions $=$ TRUE.

end if

8. Delete $s$ from NewSubsets.

end while

end while

Figure 1. Outline of basic scatter search.

Note that while this basic procedure is very aggressive in trying to improve upon the quality of the solutions in the current reference set, it those so by sacrificing search diversity. In fact, the Diversification Generation Method is used only once to generate PSize different solutions at the beginning of the search and it is never employed again. The initial RefSet is built selecting a solution from $P$ and then making $b-1$ more selection in order to maximize the minimum distance between the candidate solution and the solutions currently in RefSet. That is, for each candidate solution $x$ in P-RefSet and reference set solution $y$ in RefSet, we calculate a measure of distance or dissimilarity $d(x, y)$. We then select the candidate solution that maximizes $d_{\min }(x)$, where $d_{\min }(x)=\min _{y \in \operatorname{RefSet}}\{d(x, y)\}$.

The updating of the reference set is based on improving the quality of the worst solution and the search terminates when no new solutions are admitted to RefSet. The procedure does not use an Improvement Method, which in scatter search implementations is typically applied after a new solution is constructed with either the Diversification Generation Method or the Combination Method. The Subset Generation Method is also very simple and consists of generating all pairs of solutions in RefSet that contain at least one new solution. This means that the procedure does not allow for two solutions to be subjected to the Combination Method more than once.

In the remainder of this paper, we develop and test scatter search designs that add to the basic procedure of Figure 1. All of these variations employ a method for creating new solutions that consists of linear combinations of two solutions in the reference set. Linear combinations of two solutions were suggested by Glover (1994) in the context of nonlinear optimization and are a generalization of the linear or arithmetical crossover also used in continuous and convex spaces (Michalewicz and Logan 1994). We consider the following three types of linear combinations, where we assume that the reference solutions are $x^{\prime}$ and $x^{\prime \prime}, d=r \frac{x^{\prime \prime}-x^{\prime}}{2}$ and $r$ is a random number in the range $(0,1)$ : 


$$
\begin{array}{ll}
\mathrm{C} 1: & x=x^{\prime}-d \\
\mathrm{C} 2: & x=x^{\prime}+d \\
\mathrm{C} 3: & x=x^{\prime \prime}+d
\end{array}
$$

In the basic procedure of Figure 1, the Combination Method generates one solution of each type when combining two solutions in the reference set. Note that the combination mechanism includes a random element and therefore it is possible to combine the same pair of solutions more than once to generate new solutions. The main motivation for using a random component in the Combination Method is that it would be difficult to calibrate the procedure and find effective $r$-values for a variety of problems. The Subset Combination Method does not allow the same pair of solutions to be combined more than once.

\section{Reference Set Update Method}

In the basic procedure of Figure 1, the reference set is updated by replacing the reference solution with the worst objective function value with a new solution that has a better objective function value. Since the RefSet is always ordered, the best solution can be denoted by $x^{1}$ and worst solution by $x^{b}$. Then, when a new solution $x$ is generated as a result of the application of the Combination Method, the objective function value of the new solution is used to determine whether the RefSet needs to be updated. That is, if:

$$
x \notin \operatorname{RefSet} \text { and } f(x)<f\left(x^{b}\right)
$$

we update RefSet by making $x^{b}=x$ and reorder the reference set. We have developed the following three variations of this basic update procedure (UP0), which we will refer to as UP1, UP2 and UP3.

\section{RefSet Update UP1}

This update adds a mechanism to partially rebuild the reference set when no new solutions can be generated with the Combination Method. The update is performed after the inner "while-loop" in Figure 1 fails and the NewSolutions variable is FALSE. (The inner while-loop consists of steps 4 to 8.) The RefSet is partially rebuilt with a diversification update, which works as follows. Solutions $x^{[b / 2]+1}, \ldots, x^{b}$ are deleted from RefSet, where $[v]$ is the largest integer less than or equal to $v$. The frequency counts freq $(i, j)$, the number of times sub-range $j$ has been chosen to generate a value for variable $i$, in the Diversification Generation Method are updated with the corresponding values from the solutions that remain in the reference set, that is, $x^{1}, \ldots, x^{[b / 2]}$. Then, the generator is used to construct a set $P$ of solutions. Solutions $x^{[b / 2]+1}, \ldots, x^{b}$ in RefSet are sequentially selected from $P$ with the max-min criterion used in step 1 of Figure 1 (and described in the previous section). The min-max criterion is applied against solutions $x^{1}, \ldots, x^{[b / 2]}$ when selecting solution $x^{[b / 2]+1}$, then against solutions $x^{1}, \ldots, x^{[b / 2]+1}$ when selecting solution $x^{[b / 2]+2}$, and so on.

The following modification of the pseudo-code in Figure 1 incorporates the UP1 update. The modification consists of adding the following instructions after the end of the inner while-loop:

if $($ NewSolutions $=$ FALSE $)$ then

9. Use the Diversification Generation Method to replace solutions $x^{[b / 2]+1}, \ldots, x^{b}$ from RefSet and reorder RefSet.

10. Make NewSolutions $=$ TRUE

end if

Note that when this update is added to the basic procedure, the resulting search does not have a built-in termination criterion. In our experimental testing we stop the search after a maximum number of objective function evaluations has been reached. The basic Combination Method is applied when using the UP1 update. 


\section{RefSet Update UP2}

This update has the goal of dynamically preserving diversity in the reference set, instead of allowing it to become homogenous by only admitting high quality solutions that in many applications tend to be very similar to each other. Hence, in addition to updating the reference set when new solutions of high quality are generated, the reference set is also updated with highly diverse solutions. Specifically, the update consists of partitioning the reference into two subsets: $\operatorname{RefSet}_{1}=\left\{x^{1}, \ldots, x^{[b / 2]}\right\}$ and RefSet $_{2}=\left\{x^{[b / 2]+1}, \ldots, x^{b}\right\}$. The first subset is referred to as the "quality" subset and the second is referred to as the "diverse" subset. The solutions in RefSet $_{1}$ are ordered according to their objective function value and the set is updated with the goal of increasing quality, using the UP0 criterion. That is, a new solution $x$ replaces the reference solution $x^{[b / 2]}$ if $f(x)<f\left(x^{[b / 2]}\right)$. The solutions in RefSet $_{2}$ are ordered according to their diversity value and the update has the goal of increasing diversity. Therefore, a new solution $x$ replaces the reference solution $x^{b}$ if $d_{\min }(x)>d_{\min }\left(x^{b}\right)$.

We can modify the outline in Figure 1 to incorporate the UP2 update by replacing the "if" statement inside the inner while-loop with the following:

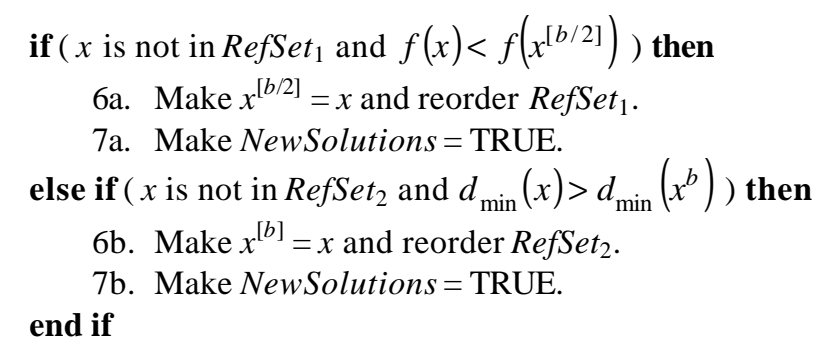

When adding this update to the basic procedure, the procedure exits the inner while -loop when no new solutions can be admitted to either RefSet R $_{1}$ RefSet $_{2}$ during the combination of all NewSubsets. The search, however, does not terminate, because RefSet is updated using the UP1 update. That is, RefSet is kept and RefSet $_{2}$ is rebuilt with the Diversification Generation Method. The following rules are used during the application of the Combination Method when the UP2 update is active, assuming that $x^{\prime}$ and $x^{\prime \prime}$ are the reference solutions to be combined:

- If both $x^{\prime}$ and $x^{\prime \prime}$ are elements of RefSet $_{1}$, then C1 and C3 are applied once and C2 is applied twice to create 4 solutions.

- If only one of $x^{\prime}$ and $x^{\prime \prime}$ is a member of $\operatorname{RefSet}_{1}$, then C1, C2 and C3 are applied once to create 3 solutions.

- If neither $x^{\prime}$ nor $x^{\prime \prime}$ is a member of $\operatorname{RefSet}_{1}$, then C2 and either C1 or C3 is applied once to create 2 solutions, where the selection of $\mathrm{C} 1$ or $\mathrm{C} 3$ is random.

The rationale behind these rules is that we have experimentally determined that high-quality solutions tend to create other high-quality solutions and therefore we allow combinations of two high-quality solutions to create more new solutions than other types of combinations (Campos, et al. 1999). Note that we do not use common random numbers during the application of these combination methods.

\section{RefSet Update UP3}

This update is an extension of UP2. The reference set is divided into three subsets: $\operatorname{RefSet}_{1}=\left\{x^{1}, \ldots, x^{[(b-}\right.$ $\left.{ }^{2) / 2]}\right\}, \operatorname{RefSet}_{2}=\left\{x^{[(b-2) / 2]+1}, \ldots, x^{b-2}\right\}$, and $\operatorname{RefSet}_{3}=\left\{x^{b-1}, x^{b}\right\} . \operatorname{RefSet}_{1}$ and $\operatorname{RefSet}_{2}$ are updated using the same rules as in UP2. In order to update $\operatorname{RefSet}_{3}$, we keep track of $g(x)$, the objective function value of the best solution ever created from a combination of $x \in \operatorname{RefSet}_{1}$ and any other reference solution. RefSet R $_{3}$ ordered according to $g(x)$ in such a way that $g\left(x^{b-1}\right)<g\left(x^{b}\right)$. When solution $x^{[(b-2) / 2]}$ in $\operatorname{RefSet}_{1}$ is replaced 
with a newly created solution of higher quality, we compare $g\left(x^{[(b-2) / 2]}\right)$ and $g\left(x^{b}\right)$ and update RefSet 3 accordingly.

We can modify the pseudo-code in Figure 1 to incorporate the UP3 update by replacing the "if" statement inside the inner while -loop with the following:

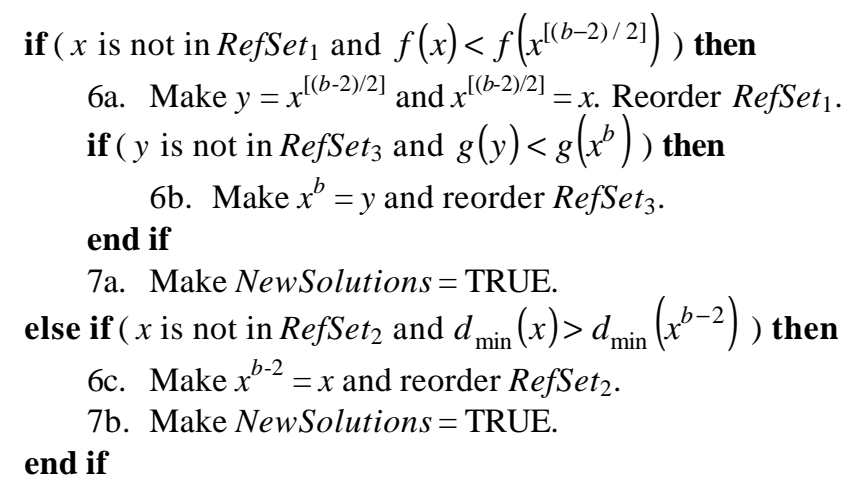

When adding this update to the basic procedure, the search exists the inner while-loop when no new solutions can be admitted to either RefSet $t_{1}$ or RefSet during the combination of all NewSubsets. As before, the search does not terminate, because RefSet is updated in a similar way as UP2. That is, RefSet $_{1}$ and RefSet $_{3}$ are kept and RefSet $_{2}$ is rebuilt with the Diversification Generation Method. Note that the update of $\operatorname{RefSet}_{3}$ is dependent on the update of $\operatorname{RefSet}_{1}$, in the sense that $\operatorname{RefSet}_{3}$ can only be updated if a solution in RefSet $_{1}$ is replaced. The combination rules associated with the UP3 update are the same as the rules for UP2 when combining solutions from either RefSet ${ }_{1}$ or RefSet $t_{2}$. However, when $x^{\prime}$ is in $\operatorname{RefSet}_{1}$ and $x^{\prime \prime}$ is in $\operatorname{RefSet}_{3}$, then $\mathrm{C} 1$ and $\mathrm{C} 2$ are applied once with $d=r \frac{x^{\prime \prime}-x^{\prime}}{3}$. Note that we don't attempt combinations that consist of either one solution from RefSet $_{2}$ and one solution from RefSet $_{3}$ or both solutions from RefSet $_{3}$.

\section{Diversification Generation Method}

The basic Diversification Generation Method (DV0) uses controlled randomization and frequency memory to generate a set of diverse solutions. We accomplish this by dividing the range of each variable $u_{i}-l_{i}$ into 4 sub-ranges of equal size. Then, a solution is constructed in two steps. First a sub-range is randomly selected. The probability of selecting a sub-range is inversely proportional to its frequency count. Then a value is randomly generated within the selected sub-range. The number of times sub-range $j$ has been chosen to generate a value for variable $i$ is accumulated in $\operatorname{freq}(i, j)$.

In addition to DV0, we have developed two variants (DV1 and DV2), which are based on techniques from the area of statistics known as Design of Experiments. One of the most popular design of experiments is the factorial design $k^{n}$, where $n$ is the number of factors (in our case variables) and $k$ is the number of levels (in our case possible variable values). A full factorial design considers that all combinations of the factors and levels will be tested. Therefore, a full factorial design with 5 factors and 3 critical levels would require $3^{5}=243$ experiments. Obviously, full factorial designs can quickly become impractical even for a small number of levels, because the number of experiments exponentially increases with the number of factors. A more practical alternative is to employ fractional factorial designs. These designs draw conclusions based on a fraction of experiments, which are strategically selected from the set of all possible experiments in the corresponding full factorial design. One of the most notable proponents of the use of fractional factorial designs is Genichi Taguchi (Roy, 1990). Taguchi proposed a special set of orthogonal arrays to lay out experiments associated with quality improvement in manufacturing. These orthogonal arrays are the result of combining orthogonal Latin squares in a unique manner. We use Taguchi's arrays as a 
mechanism for generating diversity. Table 1 shows the $\mathrm{L}_{9}\left(3^{4}\right)$ orthogonal array that can be used to generate 9 solutions for a 4 -variable problem.

Table 1. L9 $\left(3^{4}\right)$ orthogonal array.

\begin{tabular}{ccccc}
\hline Experiment & \multicolumn{4}{c}{ Factors } \\
& 1 & 2 & 3 & 4 \\
\hline 1 & 1 & 1 & 1 & 1 \\
2 & 1 & 2 & 2 & 2 \\
3 & 1 & 3 & 3 & 3 \\
4 & 2 & 1 & 2 & 3 \\
5 & 2 & 2 & 3 & 1 \\
6 & 2 & 3 & 1 & 2 \\
7 & 3 & 1 & 2 & 3 \\
8 & 3 & 2 & 1 & 3 \\
9 & 3 & 3 & 2 & 1 \\
\hline
\end{tabular}

The values in Table 1 represent the levels at which the factors are set in each experiment. For the purpose of creating DV1, a diversification generator based on Taguchi tables, we translate each level setting as follows:

$$
\begin{aligned}
& 1=\text { lower bound }\left(l_{i}\right) \\
& 2=\text { midpoint }\left(\frac{l_{i}+u_{i}}{2}\right) \\
& 3=\text { upper bound }\left(u_{i}\right)
\end{aligned}
$$

The generator DV1 uses the appropriate Taguchi table to draw solutions every time the diversification generator is called. The Taguchi table is selected according to the number of variables in the problem. When DV1 is employed and all solutions in the appropriate table have been tried, the generator returns solutions using DV0.

A second variant based on Taguchi tables (DV2) is obtained when level settings in each experiment are translated to variable values as follows:

$$
\begin{aligned}
& 1=\text { near lower bound }\left(l_{i}+r\left(u_{i}-l_{i}\right)\right), \text { where } r \text { is randomly drawn from }(0,0.1) \\
& 2=\text { near midpoint }\left(\frac{l_{i}+u_{i}}{2}+r\left(u_{i}-l_{i}\right)\right), \text { where } r \text { is randomly drawn from }(-0.1,0.1) \\
& 3=\text { near upper bound }\left(u_{i}-r\left(u_{i}-l_{i}\right)\right), \text { where } r \text { is randomly drawn from }(0,0.1)
\end{aligned}
$$

Although the same Taguchi experiment can result in more than one solution to the problem, we turn control to DV0 once all experiments have been used to generate solutions, because DV0 covers the entire feasible range of each variable, while DV2 focuses on generating solutions with variable values near the bounds and the midpoint.

\section{Intensification Strategy}

The Combination Method is the main intensification mechanism within scatter search. In section 2, we described the application of the Combination Method associated with each variant of the updating strategies for the reference set. In addition to these rules, we have developed and tested a two-phase intensification procedure.

The basic design of Figure 1 applies the Combination Method to all the subsets generated from a given reference set. Although the reference set is dynamically updated, the new solutions are not used for 
combination until all the subsets generated with the previous RefSet have been combined. In other words, the new solutions that become members of RefSet are not combined until all pairs in NewSubsets are subjected to the Combination Method. The goal of the first phase of our intensification strategy is to apply the Combination Method to new solutions in a manner that is faster than in the bas ic design. That is, if a new solution is admitted to the reference set the intensification goal is to allow this new solution to be subjected to the Combination Method as quickly as possible. This strategy can be easily implemented by modifying step 3 in Figure 1 as follows:

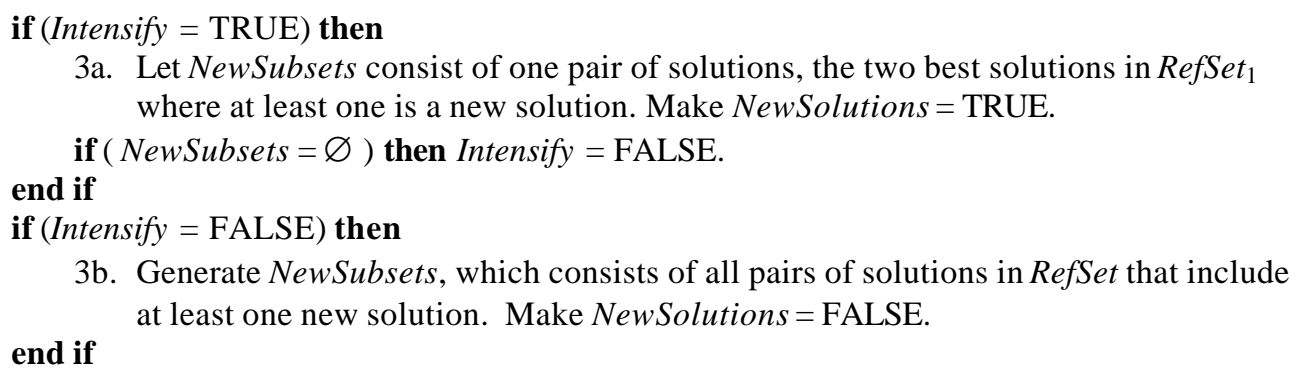

Note that by reducing NewSubsets to just one pair of solutions, the procedure returns to the generation of subsets immediately after one application of the Combination Method. The downside of this intensification phase is that some solutions in RefSet could be replaced before being combined. To illustrate this, suppose that the initial RefSet $=\left\{x^{1}, x^{2}, x^{3}, x^{4}\right\}$ and that the intensification strategy is active. Suppose also that the combination of the pair $\left(x^{1}, x^{2}\right)$ results in a solution $y$ for which:

$$
f(y)<f\left(x^{3}\right)
$$

Then, the reference set is changed in such a way that the updated RefSet $=\left\{x^{1}, x^{2}, y, x^{3}\right\}$. The search continues with the combination of the pair $\left(x^{1}, y\right)$. Clearly, the quick updating of the reference set eliminates the combination of the pair $\left(x^{1}, x^{4}\right)$.

The second phase of our intensification strategy consists of selecting the two best solutions in RefSet $_{1}$ to create 8 solutions by applying $\mathrm{C} 1$ and $\mathrm{C} 3$ twice and $\mathrm{C} 2$ four times. Note that for this intensification, we do not require that at least one of the two best solutions be new. We do not test the two phases of our intensification strategies separately. That is, we either apply the entire two-phase strategy or we don't apply it at all. The application of the intensification strategy depends on two parameters: IntPoint and IntLength. IntPoint is the iteration number at which the first intensification phase is applied for IntLength iterations. The second intensification phase is automatically applied at iteration $2 *$ IntPoint. An iteration, in our context, is considered a single evaluation of the objective function.

\section{Computational Experiments}

Our computational testing consists of two main experiments. In the first experiment, we compare the performance of the scatter search variants that result from the application of the alternative strategies described in the previous sections. In the second experiment, we compare the performance of the most effective scatter search design against a well-known genetic algorithm. Table 2 shows summary information of 40 test problems that are based on a set of nonlinear objective functions, most of which can be found in the following web pages:

$$
\begin{aligned}
& \text { http://www.maths.adelaide.edu.au/Applied/llazausk/alife/realfopt.htm } \\
& \text { http://solon.cma.univie.ac.at/ neum/glopt/my_problems.html } \\
& \text { http://www-math.cudenver.edu/ rvan/phd/node32.html }
\end{aligned}
$$

The numbers between parentheses associated with some of the problems are the parameter values for the corresponding objective function. A typical parameter refers to the number of variables, since several of these functions expand to an arbitrary number of variables. Although the objective functions are built in a 
way that the optimal solutions are known, the optimization problems cannot be trivially solved by search procedures that do not exploit the special structure associated with each function. A detailed description of the objective functions is provided in the Appendix.

Table 2. Test problems

\begin{tabular}{|c|c|c|c|c|}
\hline $\begin{array}{l}\text { Number of } \\
\text { variables }\end{array}$ & $\begin{array}{c}\text { Problem } \\
\text { number }\end{array}$ & $\begin{array}{c}\text { Name and } \\
\text { parameter values }\end{array}$ & $x$ & $f\left(x^{\prime \prime}\right)$ \\
\hline \multirow[t]{12}{*}{2} & 1 & Branin & $(9.42478,2.475) \dagger$ & 0.397887 \\
\hline & 2 & B2 & $(0,0)$ & 0 \\
\hline & 3 & Easom & $(\pi, \pi)$ & -1 \\
\hline & 4 & Goldstein and Price & $(0,-1)$ & 3 \\
\hline & 5 & Shubert & $(0.0217,-0.9527) \dagger$ & -186.7309 \\
\hline & 6 & Beale & $(3,0.5)$ & 0 \\
\hline & 7 & Booth & $(1,3)$ & 0 \\
\hline & 8 & Matyas & $(0,0)$ & 0 \\
\hline & 9 & SixHumpCamelback & $(0.089840,-0.712659) \dagger$ & -1.0316285 \\
\hline & 10 & Schwefel(2) & $(1,1)$ & 0 \\
\hline & 11 & Rosenbrock (2) & $(1,1)$ & 0 \\
\hline & 12 & Zakharov(2) & $(0,0)$ & 0 \\
\hline \multirow[t]{2}{*}{3} & 13 & De Joung & $(0,0,0)$ & 0 \\
\hline & 14 & $\operatorname{Hartmann}(3,4)$ & $(0.114614,0.555649,0.852547)$ & 0 \\
\hline \multirow[t]{7}{*}{4} & 15 & Colville & $(1,1,1,1)$ & 0 \\
\hline & 16 & Shekel(5) & $(4,4,4,4)$ & -10.1532 \\
\hline & 17 & Shekel(7) & $(4,4,4,4)$ & -10.4029 \\
\hline & 18 & Shekel(10) & $(4,4,4,4)$ & -10.53641 \\
\hline & 19 & $\operatorname{Perm}(4,0.5)$ & $(1,2,3,4)$ & 0 \\
\hline & 20 & Perm0 $(4,10)$ & $(1,1 / 2,1 / 3,1 / 4)$ & 0 \\
\hline & 21 & Powersum $(8,18,44,114)$ & $(1,2,2,3)$ & 0 \\
\hline \multirow[t]{3}{*}{6} & 22 & $\operatorname{Hartmann}(6,4)$ & $\begin{array}{c}(0.20169,0.150011,0.47687 \\
0.275332,0.311652,0.6573)\end{array}$ & 0 \\
\hline & 23 & Schwefel(6) & $(1, \ldots, 1)$ & 0 \\
\hline & 24 & $\operatorname{Trid}(6)$ & $x_{i}=i^{*}(7-i)$ & -50 \\
\hline \multirow[t]{6}{*}{10} & 25 & Trid(10) & $x_{i}=i^{*}(11-i)$ & -210 \\
\hline & 26 & Rastrigin(10) & $(0, \ldots, 0)$ & 0 \\
\hline & 27 & Griewank(10) & $(0, \ldots, 0)$ & 0 \\
\hline & 28 & Sum Squares(10) & $(0, \ldots, 0)$ & 0 \\
\hline & 29 & Rosenbrock(10) & $(1, \ldots, 1)$ & 0 \\
\hline & 30 & Zakharov(10) & $(0, \ldots, 0)$ & 0 \\
\hline \multirow[t]{5}{*}{20} & 31 & Rastrigin(20) & $(0, \ldots, 0)$ & 0 \\
\hline & 32 & Griewank(20) & $(0, \ldots, 0)$ & 0 \\
\hline & 33 & Sum Squares(20) & $(0, \ldots, 0)$ & 0 \\
\hline & 34 & Rosenbrock(20) & $(1, \ldots, 1)$ & 0 \\
\hline & 35 & Zakharov(20) & $(0, \ldots, 0)$ & 0 \\
\hline \multirow[t]{6}{*}{$>20$} & 36 & Powell(24) & $(3,-1,0,1,3, \ldots, 3,-1,0,1)$ & 0 \\
\hline & 37 & Dixon and Price(25) & $-\left(\frac{z-1}{-1}\right)$ & 0 \\
\hline & & & $x_{i}=2(\bar{z}), z=2^{i-1}$ & \\
\hline & 38 & Levy(30) & $(1, \ldots, 1)$ & 0 \\
\hline & 39 & Sphere(30) & $(0, \ldots, 0)$ & 0 \\
\hline & 40 & Ackley (30) & $(0, \ldots, 0)$ & 0 \\
\hline
\end{tabular}

$\uparrow$ This is one of several multiple optimal solutions.

Since the initial reference set includes the solution in which all the variables are set to their lower bound (i.e., $x=l$ ), the solution in which all the variables are set to their upper bounds (i.e., $x=u$ ) and the solution for which all the variables are set to the midpoint (i.e., $x=(u+l) / 2)$, we have modified the bounds in those cases where any of these solutions turns out to be the optimal. For example, the optimal solution to the "De Joung" function is $x=0$ and the original bounds for all variables were $l=-5.12$ and $u=5.12$. Since the 
midpoint of this range is the optimal solution, the problem is trivially solved by any of our scatter search variants. Therefore, we have modified the range and changed the lower bound to -2.56 .

For our experiments, we define the optimality gap as:

$$
G A P=\left|f(x)-f\left(x^{*}\right)\right|
$$

where $x$ is a heuristic solution and $x^{*}$ is the optimal solution. We then say that a heuristic solution $x$ is optimal if:

$$
G A P \leq \begin{cases}\varepsilon & f\left(x^{*}\right)=0 \\ \varepsilon *\left|f\left(x^{*}\right)\right| & f\left(x^{*}\right) \neq 0\end{cases}
$$

In our experimentation we set $\varepsilon=0.001$. Our first experiment consists of trying all possible combinations of the designs resulting from the strategies described above. We consider the following settings:

Reference set update: $\quad$ UP1, UP2 and UP3

Diversification generation: DV0, DV1 and DV2

Intensification strategy: IN0 (deactivated) and IN1 (activated)

We use IntPoint $=3000$ and IntLength $=200$ for IN1. These settings result in $3 * 3 * 2=18$ possible combinations and each combination is run up to a maximum of 10000 objective function evaluations. Note that we don't test settings with UP0 because this update strategy causes an early termination of the search and cannot compete with extended runs of the other variants. We execute $18 * 20=360$ runs of our scatter search procedure, because we limit this experiment to the odd-numbered problems in Table 2. We use half of the available problems to be able to confirm our findings when applying them to the entire set of problems. The outcome from the first experiment is summarized in Table 3.

Table 3. Results of various scatter search designs.

\begin{tabular}{cccccc}
\hline $\begin{array}{c}\text { Update Method } \\
\text { (UP) }\end{array}$ & $\begin{array}{c}\text { Diversification } \\
\text { Method (DV) }\end{array}$ & $\begin{array}{r}\text { Intensification } \\
\text { Strategy (IN) }\end{array}$ & Average GAP & Std. Dev. GAP & $\begin{array}{c}\text { Number of } \\
\text { Optima }\end{array}$ \\
\hline 1 & 0 & 0 & 9.90 & 30.93 & 14 \\
1 & 0 & 1 & 6.14 & 26.44 & 13 \\
1 & 1 & 0 & 45.76 & 171.00 & 13 \\
1 & 1 & 1 & 10.76 & 32.21 & 14 \\
1 & 2 & 0 & 35.84 & 127.98 & 7 \\
1 & 2 & 1 & 34.07 & 117.99 & 9 \\
2 & 0 & 0 & 46.15 & 177.96 & 8 \\
2 & 0 & 1 & 45.94 & 178.01 & 6 \\
2 & 1 & 0 & 68.89 & 208.02 & 6 \\
2 & 1 & 1 & 55.58 & 131.76 & 3 \\
2 & 2 & 0 & 59.62 & 193.65 & 1 \\
2 & 2 & 1 & 48.00 & 116.76 & 2 \\
3 & 0 & 0 & 40.47 & 150.91 & 8 \\
3 & 0 & 1 & 47.82 & 183.01 & 9 \\
3 & 1 & 0 & 69.82 & 206.21 & 3 \\
3 & 1 & 1 & 77.90 & 206.01 & 5 \\
3 & 2 & 0 & 45.53 & 121.89 & 5 \\
3 & 2 & 1 & 47.02 & 122.59 & 2 \\
\hline
\end{tabular}


The results in Table 3 indicate that there is an important difference between the number of optimal solutions found with the (UP1, DV0, *) and (UP1, DV1, *) settings and all other settings tested in our first experiment. Among the top four settings, the (UP1, DV0, IN1) seems to dominate the others in terms of the average $G A P$.

Since the GAP variability is quite large with respect to the average values, we conducted an analysis of variance to detect statistically significant differences. Table 4 shows the analysis of variance output from SPSS. The model tests for differences in means of the dependent variable GAP and includes the three factors UP, DV and IN.

Table 4. ANOVA for scatter search designs.

\begin{tabular}{lrrrrr}
\hline \multicolumn{1}{c}{ Source } & $\begin{array}{c}\text { Sum of } \\
\text { Squares }\end{array}$ & $\mathrm{df}$ & Mean Square & $\mathrm{F}$ & $p$-value \\
\hline UP & 75196.848 & 2 & 37598.424 & 1.657 & 0.192 \\
DV & 29293.122 & 2 & 14646.561 & 0.645 & 0.525 \\
IN & 2641.153 & 1 & 2641.153 & 0.116 & 0.733 \\
UP * DV & 13664.106 & 4 & 3416.026 & 0.151 & 0.963 \\
UP * IN & 5892.651 & 2 & 2946.325 & 0.130 & 0.878 \\
DV * IN & 3265.096 & 2 & 1632.548 & 0.072 & 0.931 \\
UP * DV * IN & 4959.722 & 4 & 1239.930 & 0.055 & 0.994 \\
Error & 7761017.193 & 342 & 22693.033 & & \\
Total & 8598536.376 & 360 & & & \\
\hline
\end{tabular}

The $p$ values in Table 4 are clearly larger than any reasonable critical value that one might use to test the significance of the mean GAP. Therefore the ANOVA in Table 4 indicates that the mean GAP does not differ more than would be expected by chance alone.

In our second main experiment, we compare the performance of our scatter search with UP1, DV0 and IN1 against Genocop III (http://www.coe.uncc.edu/ zbyszek/gchome.html), an implementation of genetic algorithms that is customized for solving nonlinear optimization problems with continuous and bounded variables (Michalewicz 1993). We chose the (UP1, DV0, IN1) setting because it is the one with the smallest average GAP in Table 3 and also is one of four with the largest number of optima found.

Table 5 shows the average GAP value for our scatter search implementation compared to the one for Genocop III. The average GAP is calculated over the set of 40 problems in Table 2 at several points during the search. Since Genocop III begins with a GAP in the order of $10^{27}$ for problem 23 , we also provide the average $G A P$ calculated over 39 problems, ignoring the GAP for problem 23.

Table 5. Average GAP values.

\begin{tabular}{lrrrrrrr}
\hline Evaluations & \multicolumn{1}{c}{100} & \multicolumn{1}{c}{500} & \multicolumn{1}{c}{1000} & \multicolumn{1}{c}{5000} & \multicolumn{1}{c}{10000} & \multicolumn{1}{c}{20000} & 50000 \\
\hline Genocop $^{1}$ & $5.37 \mathrm{E}+25$ & $2.39 \mathrm{E}+17$ & $1.13 \mathrm{E}+14$ & 636.37 & 399.52 & 320.84 & 313.34 \\
Genocop $^{2}$ & 1335.45 & 611.30 & 379.03 & 335.81 & 328.66 & 324.72 & 321.20 \\
Scatter Search $^{2}$ & 134.45 & 26.34 & 14.66 & 4.96 & 3.60 & 3.52 & 3.46 \\
\hline
\end{tabular}

${ }^{1}$ Average values over all test problems.

${ }^{2}$ Average values ignoring problem 23.

Table 5 shows that the average GAP of scatter search is consistently lower than the average GAP associated with Genocop III. In fact, the average GAP of scatter search after 100 evaluations of the objective function is already better than the average GAP for Genocop III after 50000 objective function evaluations. The final GAP values for scatter search are all less than 1.0, except for problems $23,26,34$, and 40, for which the $G A P$ values are $118.4341,9.9496,2.2441$, and 5.5033, respectively. Although there is a difference in average GAP values in Table 5 between scatter search and Genocop, an analysis of variance revealed that the difference of means is not statistically significance. SPSS yields a $p$-value of 0.164 for the comparison of means at 5000 evaluations with a one-way ANOVA. 
Counting the number of optimal solutions found with each method is an alternative measure of performance, as shown in Table 3. The plot in Figure 2 shows the number of $\varepsilon$-optimal solutions found by each procedure during a search with a stopping criterion of 50000 objective function evaluations. The plot shows the better performance of the scatter search implementation, which solves 4 problems within 100 evaluations and 30 by 20000 evaluations. At the end of the search, Genocop III successfully solves a total of 23 problems.

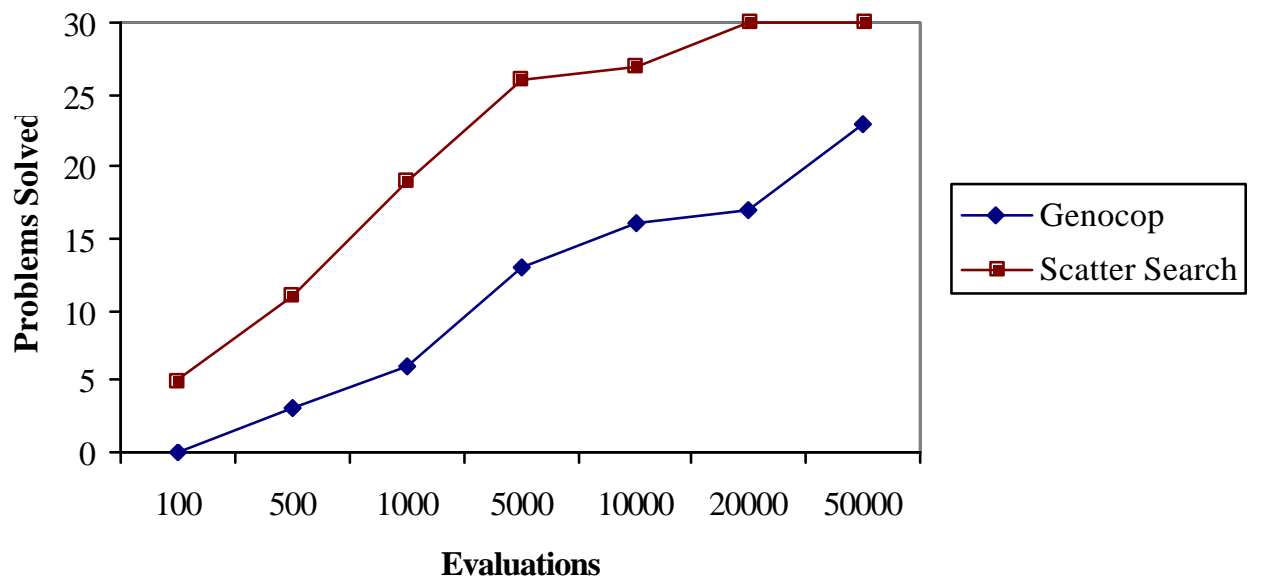

Figure 2. Number of problems solved.

We perform a secondary experiment to investigate whether any of the scatter search variants is capable of solving the 10 problems that the UP1, DV0 and IN1 setting did not solve. For each unsolved problem (15, $19,23,26,29,34,36-38$ and 40 ), we run a 50000-evaluation search using each of the 17 settings, excluding (UP1, DV0, IN1). The outcome of this experiment is that 5 additional problems are solved and the average GAP of the unsolved problems is reduced to 1.2322. These results are summarized in Tables 6 and 7.

Table 6 shows that both settings (UP1, DV1, IN0) and (UP1, DV1, IN1) are capable of solving problems 15,19 and 26. However, the performance of (UP1, DV1, IN0) is considered better regarding these three problems because it solves them in fewer evaluations than (UP1, DV1, IN1). This setting, on the other hand, is capable of solving the elusive problem 23 employing less than 10000 evaluations. Also, a setting that uses UP1 and DV0 solves problem 36 regardless of the intensification strategy. Finally, note that from the unsolved problems in Table 7, only two have a GAP value larger than 1.

Table 6. Additional solved problems.

\begin{tabular}{lcc}
\hline Setting & Problem & Evaluations \\
\hline (UP1, DV1, IN0) & 15 & 13638 \\
& 19 & 13325 \\
& 26 & 27 \\
(UP1, DV1, IN1) & 15 & 16152 \\
& 19 & 37118 \\
& 23 & 9525 \\
& 26 & 27 \\
(UP2, DV0, *) & 36 & 119 \\
\hline
\end{tabular}

$*=$ all options. 
Table 7. Unsolved problems.

\begin{tabular}{clc}
\hline Problem & \multicolumn{1}{c}{ Setting } & GAP \\
\hline 29 & (UP1, DV2, IN1) & 0.327761 \\
34 & (UP1, DV1, IN1) & 2.050292 \\
37 & (UP1, *, *) & 0.666667 \\
38 & (UP1, DV0, IN1) & 0.089528 \\
40 & (UP1, DV2, IN1) & 3.026937 \\
\hline Average & & 1.232237 \\
\hline * all options.
\end{tabular}

The comparisons are made on the basis of solution quality versus number of evaluations because computing times are equivalent for all the tested methods. For instance, the average time to complete a run of 20000 objective function evaluations is 0.4 seconds for both SS (UP1, DV0, IN1) and Genocop on a $2.53 \mathrm{GHz}$ Pentium 4 computer. The use of number of function evaluations as a way of comparing procedures has the additional advantage of providing a more accurate performance measure in settings where the evaluation of the objective function is computationally expensive. For example, in the context of optimizing simulations, the time required for generating solutions is negligible compared to the time required to evaluate the objective function.

\section{Conclusions}

We have explored alternative mechanisms to perform key operations within the scatter search framework. In particular, we have focused on designing and testing strategies for updating the reference set, generating diversity and intensifying the search. We have gathered a set of 40 test problems with number of variables ranging from 2 to 30 to perform experiments with the goal of assessing the merit of each combination of the proposed strategies. For our initial testing, we chose 20 problems out of 40 and concluded that the best parameter setting was UP1, DV0 and IN1. This conclusion is based on the average GAP value and the number of optimal solutions found, although an ANOVA could not confirm a statistical significant difference. We then used this setting to compare the performance of the resulting procedure against a wellknown genetic algorithm. The computational tests show that our scatter search is robust, because it finds solutions of reasonable quality from the beginning of the search. This is an important feature in settings where the objective function evaluation is computational expensive (e.g., when optimizing simulations). The procedure is capable of finding $\varepsilon$-optimal solutions to 30 out of 40 problems within 20000 objective function evaluations. This compares favorable to the number of $\varepsilon$-optimal solutions found with Genocop III employing more than twice the number of evaluations.

\section{References}

Campos, V., F. Glover, M. Laguna and R. Martí (1999) “An Experimental Evaluation of a Scatter Search for the Linear Ordering Problem,” Working paper, University of Colorado at Boulder.

Glover, F. (1994) “Tabu Search for Nonlinear and Parametric Optimization (with Links to Genetic Algorithms)," Discrete Applied Mathematics, vol. 49, pp. 231-255.

Glover, F. (1998) “A Template for Scatter Search and Path Relinking," in Artificial Evolution, Lecture Notes in Computer Science 1363, J.-K. Hao, E. Lutton, E. Ronald, M. Schoenauer and D. Snyers (Eds.), Springer-Verlag, pp. 13-54.

Glover, F., M. Laguna and R. Martí (1999) "Scatter Search," to appear in Theory and Applications of Evolutionary Computation: Recent Trends, A. Ghosh and S. Tsutsui (Eds.), Springer-Verlag. 
Laguna, M. (2002) "Scatter Search," in Handbook of Applied Optimization, P. M. Pardalos and M. G. C. Resende (Eds.), Oxford University Press, New York, pp. 183-193.

Michalewicz, Z. (1994) Genetic Algorithms + Data Structures = Evolution Programs, Second-Extended Edition, Springer-Verlag.

Michalewicz, Z. and T. D. Logan (1994) "Evolutionary Operators for Continuous Convex Parameter Spaces," Proceedings of the $3^{\text {rd }}$ Annual Conference on Evolutionary Programming, A.V. Sebald and L. J. Fogel (Eds.), World Scientific Publishing, River Edge, NJ, pp. 84-97.

Roy, R.K. (1990) A Primer on the Taguchi Method, Van Nostrand Reinhold, New York.

\section{Appendix}

This appendix contains the description of the set of test functions in Table 2. The description consists of the objective function, parameter values and the bounds for each variable.

1. Branin

Minimize $\quad f(x)=\left(x_{2}-\left(\frac{5}{4 \pi^{2}}\right) x_{1}^{2}+\left(\frac{5}{\pi}\right) x_{1}-6\right)^{2}+10\left(1-\frac{1}{8 \pi}\right) \cos \left(x_{i}\right)+10$

Subject to $\quad-5 \leq x_{1}, x_{2} \leq 15$

2. B2

Minimize $f(x)=x_{1}^{2}+2 x_{2}^{2}-0.3 \cos \left(3 \pi x_{1}\right)-0.4 \cos \left(4 \pi x_{2}\right)+0.7$

Subject to $\quad-50 \leq x_{i} \leq 100 \quad$ for $i=1,2$

3. Easom

Minimize $\quad f(x)=-\cos \left(x_{1}\right) \cos \left(x_{2}\right) \exp \left(-\left(\left(x_{1}-\pi\right)^{2}+\left(x_{2}-\pi\right)^{2}\right)\right)$

Subject to $-100 \leq x_{i} \leq 100$ for $i=1,2$

4. Goldstein and Price

$$
\begin{aligned}
\text { Minimize } \quad f(x)= & \left(1+\left(x_{1}+x_{2}+1\right)^{2}\left(19-14 x_{1}+3 x_{1}^{2}-14 x_{2}+6 x_{1} x_{2}+3 x_{2}^{2}\right)\right) \\
& \left(30+\left(2 x_{1}-3 x_{2}\right)^{2}\left(18-32 x_{1}+12 x_{1}^{2}+48 x_{2}-36 x_{1} x_{2}+27 x_{2}^{2}\right)\right)
\end{aligned}
$$

Subject to $\quad-2 \leq x_{i} \leq 2 \quad$ for $i=1,2$

5. Shubert

$$
\text { Minimize } f(x)=\left(\sum_{j=1}^{5} j \cos \left((j+1) x_{1}+j\right)\right)\left(\sum_{j=1}^{5} j \cos \left((j+1) x_{2}+j\right)\right)
$$

Subject to $\quad-10 \leq x_{i} \leq 10 \quad$ for $i=1,2$ 
Minimize $f(x)=\left(1.5-x_{1}+x_{1} x_{2}\right)^{2}+\left(2.25-x_{1}+x_{1} x_{2}^{2}\right)^{2}+\left(2.625-x_{1}+x_{1} x_{2}^{3}\right)^{2}$

Subject to $\quad-4.5 \leq x_{1}, x_{2} \leq 4.5$

7. Booth

Minimize $f(x)=\left(x_{1}+2 x_{2}-7\right)^{2}+\left(2 x_{1}+x_{2}-5\right)^{2}$

Subject to $\quad-10 \leq x_{1}, x_{2} \leq 10$

8. Matyas

Minimize $\quad f(x)=0.26\left(x_{1}^{2}+x_{2}^{2}\right)-0.48 x_{1} x_{2}$

Subject to $\quad-5 \leq x_{1}, x_{2} \leq 10$

9. SixHumpCamelBack

Minimize $\quad f(x)=4 x_{1}^{2}-2.1 x_{1}^{4}+\frac{1}{3} x_{1}^{6}+x_{1} x_{2}-4 x_{2}^{2}+4 x_{2}^{4}$

Subject to $\quad-5 \leq x_{1}, x_{2} \leq 5$

10, 23. Schwefel $(n)$

Minimize $\quad f(x)=418.9829 n+\sum_{i=1}^{n}\left(-x_{i} \sin \sqrt{\left|x_{i}\right|}\right)$

Subject to $\quad-500 \leq x_{i} \leq 500$ for $i=1, \ldots, n$

11, 29, 34. Rosenbrock $(n)$

Minimize $\quad f(x)=\sum_{i=1}^{n / 2} 100\left(x_{2 i}-x_{2 i-1}^{2}\right)^{2}+\left(1-x_{2 i-1}\right)^{2}$

Subject to $\quad-10 \leq x_{i} \leq 10 \quad$ for $i=1, \ldots, n$.

12, 30, 35. Zakharov(n)

Minimize $\quad f(x)=\sum_{j=1}^{n} x_{j}^{2}+\left(\sum_{j=1}^{n} 0.5 j x_{j}\right)^{2}+\left(\sum_{j=1}^{n} 0.5 j x_{j}\right)^{4}$

Subject to $\quad-5 \leq x_{i} \leq 10 \quad$ for $i=1, \ldots, n$

13. De Joung

$\begin{array}{ll}\text { Minimize } & f(x)=x_{1}^{2}+x_{2}^{2}+x_{3}^{2} \\ \text { Subject to } & -2.56 \leq x_{i} \leq 5.12 \text { for } i=1,2,3\end{array}$


14. Hartmann $(3,4)$

\begin{tabular}{|c|c|c|c|c|c|c|c|}
\hline Subject to & $0 \leq x_{i} \leq 1$ & \multicolumn{3}{|c|}{ for $i=1,2,3$} & & & \\
\hline$i$ & \multicolumn{3}{|c|}{$a_{i j}$} & $c_{i}$ & \multicolumn{3}{|c|}{$p_{i j}$} \\
\hline 1 & 3.0 & 10.0 & 30.0 & 1.0 & 0.3689 & 0.1170 & 0.2673 \\
\hline 2 & 0.1 & 10.0 & 35.0 & 1.2 & 0.4699 & 0.4387 & 0.7470 \\
\hline 3 & 3.0 & 10.0 & 30.0 & 3.0 & 0.1091 & 0.8732 & 0.5547 \\
\hline 4 & 0.1 & 10.0 & 35.0 & 3.2 & 0.0381 & 0.5743 & 0.8828 \\
\hline
\end{tabular}

15. Colville

$\begin{aligned} \text { Minimize } \quad f(x)= & 100\left(x_{2}-x_{1}^{2}\right)^{2}+\left(1-x_{1}\right)^{2}+90\left(x_{4}-x_{3}^{2}\right)^{2}+\left(1-x_{3}\right)^{2}+ \\ & 10.1\left(\left(x_{2}-1\right)^{2}+\left(x_{4}-1\right)^{2}\right)+19.8\left(x_{2}-1\right)\left(x_{4}-1\right)\end{aligned}$

Subject to $\quad-10 \leq x_{i} \leq 10 \quad$ for $i=1, \ldots, 4$.

16-18. Shekel $(n)$

Minimize $\quad f(x)=-\sum_{i=1}^{n}\left(\left(\boldsymbol{x}-\boldsymbol{a}_{i}\right)^{T}\left(\boldsymbol{x}-\boldsymbol{a}_{i}\right)+c_{i}\right)^{-1} ; \boldsymbol{x}=\left(x_{1}, x_{2}, x_{3}, x_{4}\right)^{T} ; \boldsymbol{a}_{i}=\left(a_{i}^{1}, a_{i}^{2}, a_{i}^{3}, a_{i}^{4}\right)^{T}$

Subject to $\quad 0 \leq x_{i} \leq 10 \quad$ for $i=1,2,3,4$

\begin{tabular}{cccccc}
\hline$i$ & \multicolumn{5}{c}{$a_{i}^{T}$} \\
\hline 1 & 4.0 & 4.0 & 4.0 & 4.0 & $c_{i}$ \\
2 & 1.0 & 1.0 & 1.0 & 1.0 & 0.1 \\
3 & 8.0 & 8.0 & 8.0 & 8.0 & 0.2 \\
4 & 6.0 & 6.0 & 6.0 & 6.0 & 0.2 \\
5 & 3.0 & 7.0 & 3.0 & 7.0 & 0.4 \\
6 & 2.0 & 9.0 & 2.0 & 9.0 & 0.6 \\
7 & 5.0 & 5.0 & 3.0 & 3.0 & 0.3 \\
8 & 8.0 & 1.0 & 8.0 & 1.0 & 0.7 \\
9 & 6.0 & 2.0 & 6.0 & 2.0 & 0.5 \\
10 & 7.0 & 3.6 & 7.0 & 3.6 & 0.5 \\
\hline
\end{tabular}

19. $\operatorname{Perm}(n, \beta)$

Minimize $\quad f(x)=\sum_{k=1}^{n}\left(\sum_{i=1}^{n}\left(i^{k}+\beta\right)\left(\left(\frac{x_{i}}{i}\right)^{k}-1\right)\right)^{2}$

Subject to $\quad-n \leq x_{i} \leq n \quad$ for $i=1, \ldots, n$. 
20. $\operatorname{Perm} 0(n, \beta)$

Minimize $\quad f(x)=\sum_{k=1}^{n}\left(\sum_{i=1}^{n}(i+\beta)\left(x_{i}^{k}-\left(\frac{1}{i}\right)^{k}\right)\right)^{2}$

Subject to $\quad-n \leq x_{i} \leq n \quad$ for $i=1, \ldots, n$.

21. PowerSum $\left(b_{1}, \ldots, b_{n}\right)$

Minimize $f(x)=\sum_{k=1}^{n}\left(\left(\sum_{i=1}^{n} x_{i}^{k}\right)-b_{k}\right)^{2}$

Subject to $\quad 0 \leq x_{i} \leq n \quad$ for $i=1, \ldots, n$.

\section{2. $\operatorname{Hartmann}(6,4)$}

Minimize $f(x)=-\sum_{i=1}^{4} c_{i} \exp \left(-\sum_{j=1}^{6} a_{i j}\left(x_{j}-p_{i j}\right)^{2}\right)$

Subject to $\quad 0 \leq x_{i} \leq 1 \quad$ for $i=1, \ldots, 6$

\begin{tabular}{cccccccccccccc}
\hline$i$ & \multicolumn{4}{c}{$a_{i j}$} & \multicolumn{3}{c}{$c_{i}$} & \multicolumn{6}{c}{$p_{i j}$} \\
\hline 1 & 10.0 & 3.0 & 17.0 & 3.5 & 1.7 & 8.0 & 1.0 & 0.1312 & 0.1696 & 0.5569 & 0.0124 & 0.8283 & 0.5886 \\
\hline 2 & 0.05 & 10.0 & 17.0 & 0.10 & 8.0 & 14.0 & 1.2 & 0.2329 & 0.4135 & 0.8307 & 0.3736 & 0.1004 & 0.9991 \\
\hline 3 & 3.0 & 3.5 & 1.7 & 10.0 & 17.0 & 8.0 & 3.0 & 0.2348 & 0.1451 & 0.3522 & 0.2883 & 0.3047 & 0.6650 \\
\hline 4 & 17.0 & 8.0 & 0.05 & 10.0 & 0.1 & 14.0 & 3.2 & 0.4047 & 0.8828 & 0.8732 & 0.5743 & 0.1091 & 0.0381 \\
\hline
\end{tabular}

\section{4-25. Trid $(n)$}

Minimize $\quad f(x)=\left(\sum_{i=1}^{n}\left(x_{i}-1\right)^{2}\right)-\sum_{i=2}^{n} x_{i} x_{j}$

Subject to $\quad-n^{2} \leq x_{i} \leq n^{2} \quad$ for $i=1, \ldots, n$.

26, 31. Rastrigin $(n)$

Minimize $\quad f(x)=10 n+\sum_{i=1}^{n}\left(x_{i}^{2}-10 \cos \left(2 \pi x_{i}\right)\right)$

Subject to $\quad-2.56 \leq x_{i} \leq 5.12$ for $i=1, \ldots, n$

\section{7, 32. Griewank $(n)$}

Minimize $\quad f(x)=\sum_{i=1}^{n} \frac{x_{i}^{2}}{4000}-\prod_{i=1}^{n} \cos \left(\frac{x_{i}}{\sqrt{i}}\right)+1$

Subject to $\quad-300 \leq x_{i} \leq 600 \quad$ for $i=1, \ldots, n$ 


\section{8, 33. Sum Squares $(n)$}

Minimize $\quad f(x)=\sum_{i=1}^{n} i x_{i}^{2}$

Subject to $\quad-5 \leq x_{i} \leq 10 \quad$ for $i=1, \ldots, n$

36. Powell $(n)$

Minimize $\quad f(x)=\sum_{j=1}^{n / 4}\left(x_{4 j-3}+10 x_{4 j-2}\right)^{2}+5\left(x_{4 j-1}-x_{4 j}\right)^{2}+\left(x_{4 j-2}-2 x_{4 j-1}\right)^{4}+10\left(x_{4 j-3}-x_{4 j}\right)^{4}$

Subject to $\quad-4 \leq x_{i} \leq 5 \quad$ for $i=1, \ldots, n$

37. Dixon and Price $(n)$

Minimize $\quad f(x)=\sum_{i=1}^{n} i\left(2 x_{i}^{2}-x_{i-1}\right)^{2}+\left(x_{1}-1\right)^{2}$

Subject to $\quad-10 \leq x_{i} \leq 10 \quad$ for $i=1, \ldots, n$

38. $\operatorname{Levy}(n)$

Minimize $\quad f(x)=\sin ^{2}\left(\pi y_{1}\right)+\sum_{i=1}^{k-1}\left(y_{i}-1\right)^{2}\left(1+10 \sin ^{2}\left(\pi y_{i}+1\right)\right)+\left(y_{k}-1\right)^{2}\left(1+\sin ^{2}\left(2 \pi x_{k}\right)\right)$

Subject to $\quad y_{i}=1+\frac{x_{i}-1}{4} \quad$ for $i=1, \ldots, n$

$-10 \leq x_{i} \leq 10 \quad$ for $i=1, \ldots, n$

39. $\operatorname{Sphere}(n)$

$\begin{array}{ll}\text { Minimize } & f(x)=\sum_{i=1}^{n} x_{i}^{2} \\ \text { Subject to } & -2.56 \leq x_{i} \leq 5.12 \text { for } i=1, \ldots, n\end{array}$

40. Ackley $(n)$

Minimize $f(x)=20+e-20 e^{-0.2 \sqrt{\frac{1}{n} \sum_{i=1}^{n} x_{i}^{2}}}-e^{\frac{1}{n} \sum_{i=1}^{n} \cos \left(2 \pi x_{i}\right)}$

Subject to $\quad-15 \leq x_{i} \leq 30 \quad$ for $i=1, \ldots, n$ 